\title{
Modelling the potential spatial distribution of mosquito species using three different techniques
}

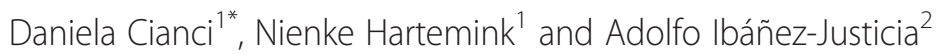

\begin{abstract}
Background: Models for the spatial distribution of vector species are important tools in the assessment of the risk of establishment and subsequent spread of vector-borne diseases. The aims of this study are to define the environmental conditions suitable for several mosquito species through species distribution modelling techniques, and to compare the results produced with the different techniques.

Methods: Three different modelling techniques, i.e., non-linear discriminant analysis, random forest and generalised linear model, were used to investigate the environmental suitability in the Netherlands for three indigenous mosquito species (Culiseta annulata, Anopheles claviger and Ochlerotatus punctor). Results obtained with the three statistical models were compared with regard to: (i) environmental suitability maps, (ii) environmental variables associated with occurrence, (iii) model evaluation.
\end{abstract}

Results: The models indicated that precipitation, temperature and population density were associated with the occurrence of Cs. annulata and An. claviger, whereas land surface temperature and vegetation indices were associated with the presence of Oc. punctor. The maps produced with the three different modelling techniques showed consistent spatial patterns for each species, but differences in the ranges of the predictions. Non-linear discriminant analysis had lower predictions than other methods. The model with the best classification skills for all the species was the random forest model, with specificity values ranging from 0.89 to 0.91 , and sensitivity values ranging from 0.64 to 0.95 .

Conclusions: We mapped the environmental suitability for three mosquito species with three different modelling techniques. For each species, the maps showed consistent spatial patterns, but the level of predicted environmental suitability differed; NLDA gave lower predicted probabilities of presence than the other two methods. The variables selected as important in the models were in agreement with the existing knowledge about these species. All model predictions had a satisfactory to excellent accuracy; best accuracy was obtained with random forest. The insights obtained can be used to gain more knowledge on vector and non-vector mosquito species. The output of this type of distribution modelling methods can, for example, be used as input for epidemiological models of vector-borne diseases.

Keywords: Species distribution modelling, Non-linear discriminant analysis, Random forest, Generalised linear model, Indigenous mosquito species, Vector-borne diseases

\footnotetext{
*Correspondence: d.cianci@uu.nl

${ }^{1}$ Faculty of Veterinary Medicine, Utrecht University, Utrecht, The Netherlands

Full list of author information is available at the end of the article
} 


\section{Background}

Mosquitoes (Diptera:Culicidae) are known to be vectors of a large number of pathogens around the globe. Bloodfeeding females of several mosquito species are involved in transmission of protozoa (e.g. Plasmodium), nematodes and viruses. Mosquitoes are considered as prime candidates for transmitting (re-)emerging vector-borne diseases in Europe [1].

Accurate information on the spatial distribution of mosquito species is essential for our understanding of the current risk of diseases transmitted by mosquitoes and for preparing for future threats [2]. For the modelling of the spatial distribution of species, several techniques exist $[3,4]$, differing in assumptions and predictive performance. The general idea behind species distribution modelling is to identify relationships between known occurrence of a species (presence/absence) and environmental data (e.g. meteorological data, land use covers, remote sensing data) and to use these relationships to make predictions for all unsampled areas in the study region.

Here, we compare non-linear discriminant analysis (NLDA), random forest (RF) and generalised linear models (GLM), three techniques that have not been compared before, by applying them to a new dataset consisting of systematically collected data on three indigenous mosquito species in the Netherlands. The three mosquito species are Culiseta annulata (Schrank, 1776), Anopheles claviger (Meigen, 1804) and Ochlerotatus punctor (Kirby, 1837). For each of these species, the resulting environmental suitability maps and the most important environmental variables selected by the models are discussed and the techniques are compared in terms of model performance.

\section{Results}

The environmental suitability for Culiseta annulata, Anopheles claviger and Ochlerotatus punctor was investigated in the Netherlands using three statistical models, i.e., NLDA, RF and GLM. Through these modelling techniques, occurrence data collected in 766 locations were linked to environmental factors.

The maps in Figure 1 show the observed occurrence data (i.e., the model input) and the predicted environmental suitability (i.e., the model outcomes). The ten most important variables for each model and each species are reported in Table 1. Accuracy measures are given in Table 2. For each species, the environmental suitability maps, the most important environmental variables and the model performances are reported below.

\section{Culiseta annulata}

Cs. annulata has the highest number of observed presences (438, Table 3) in the study. The presence points -indicated by black dots in Figure 1- show that this species was found almost all over the country. The environmental suitability maps show greater suitability in the western part of the Netherlands and lower suitability more inland. Although the areas identified as suitable (and unsuitable) are similar in all maps (Figure 1), the environmental suitability indicator for NLDA has a wider range of values (0.07-0.91) than RF (0.12-0.79) and GLM (0.10-0.86). This is visible in the different intensities of blue and red in the maps. RF and GLM identify also the northern and central part of the country as suitable environment. In all three models, precipitation and land surface temperature are important variables (Table 1). Population density comes up as an important variable in both the NLDA model and the RF model (first and second-most important variable, respectively). Highly forested areas (e.g., National Park Hoge Veluwe situated in the centre of the country) are not identified as suitable for this species in any of the three models. RF is the most specific method (specificity $=0.89$, Table 2 ), while GLM is the most sensitive (sensitivity $=0.75$, Table 2 ).

\section{Anopheles claviger}

The number of presence locations for An. claviger was 127 (Table 3). This species does not show a particular pattern in the distribution over the country (black dots in Figure 1). All maps (Figure 1) show lower environmental suitability for this species in the northern and central part of the country whereas the environment is identified as more suitable in the eastern part and in the coastal area (especially for RF and GLM). NLDA predicts much lower suitability values than the other techniques (minimum values: $\mathrm{NLDA}=0.07, \mathrm{RF}=0.22, \mathrm{GLM}=0.17$ ), only a few values are larger than 0.5 and as a consequence the average value is very low (average values: NLDA $=0$. $27, \mathrm{RF}$ and $\mathrm{GLM} \approx 0.50$ ). The GLM map indicates wetlands and floodplains as suitable environments. The most important variables are precipitation, land surface temperature, vegetation indices and middle infra-red, which is a vegetation related index (Table 1). Highly forested areas are not identified as suitable for this species. RF has excellent classification capabilities, the highest when compared to the other techniques (specificity $=0.91$, sensitivity $=0.89$, Table 2 ).

\section{Ochlerotatus punctor}

Among the three species presented here, Oc. punctor is the least present (73 presence locations, Table 3 ) and it is the only species showing a clear pattern in the observations (Figure 1); there are more presence points in the east of the country (inland). All three models indicated higher environmental suitability in this part of the country (Figure 1). Comparing the suitability values obtained with the different techniques, the values are higher for RF and GLM and lower for NLDA (maximum values: $\mathrm{NLDA}=0.81, \mathrm{RF}=0.94, \mathrm{GLM}=0.92)$. In the top 10 


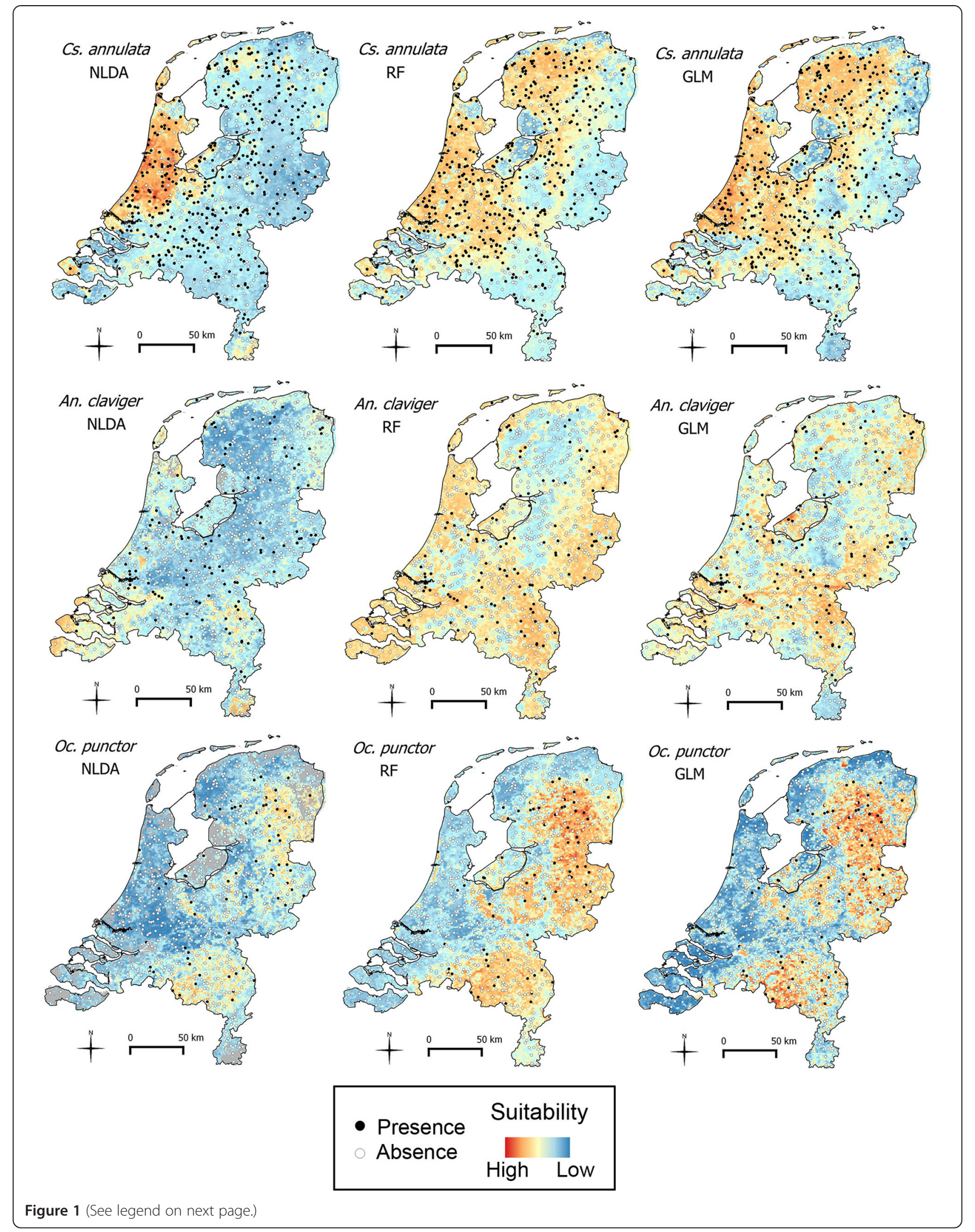


(See figure on previous page.)

Figure 1 Environmental suitability maps. Environmental suitability maps for Cs. annulata, An. claviger and Oc. punctor, produced using non-linear discriminant analysis (NLDA), random forest (RF) and generalised linear model (GLM). Black dots indicate that the species was captured on the sampled locations and white dots indicate that the species was not captured. Environmental suitability is depicted using a gradient fill: blue indicates low environmental suitability, red indicates high suitability. NLDA and GLM bootstrapping was based on 150 presence points and 150 absence points for Cs. annulata and 100 presence points and 100 absence points for An. claviger and Oc. punctor.

variables, middle-infra red, vegetation indices, precipitation and day land surface temperature are reported (Table 1). Population density is recorded as the most influential variable in NLDA and RF models. Highly forested areas are identified as suitable for this species in all three models. RF showed excellent classification capabilities (specificity $=0.91$, sensitivity $=0.95$, Table 2 ), and it is the modelling technique with the best accuracy.

\section{Discussion}

The environmental suitability for Cs. annulata, An. claviger and Oc. punctor has been investigated using field and

Table 1 Most important variables per species and per model

\begin{tabular}{|c|c|c|c|}
\hline SPECIES & NLDA & RF & GLM \\
\hline \multirow[t]{11}{*}{ Cs. annulata } & - Population density & $\cdot$ NLST P2 & • EVI VR \\
\hline & - WORLDCLIM precipitation P2 & - Population density & $\cdot$ DEM \\
\hline & - WORLDCLIM precipitation A0 & MIR A2 & - DLST A2 \\
\hline & - WORLDCLIM precipitation D1 & - DLST A2 & $\cdot$ NLST P3 \\
\hline & - WORLDCLIM precipitation DA & - MIR MX & - CMORPH precipitation VR \\
\hline & - CMORPH precipitation A1 & - NDVI A2 & - CMORPH precipitation A3 \\
\hline & - DLST DA & - MIR P1 & • DLST D1 \\
\hline & $\cdot$ DLST P1 & $\cdot \mathrm{EVI} M N$ & • DLST D3 \\
\hline & $\cdot$ DLST AO & - CMORPH precipitation P1 & - MIR A2 \\
\hline & - DLST P2 & - WORLDCLIM precipitation P1 & - MIR 03 \\
\hline & & - DLST A3 & \\
\hline \multirow[t]{10}{*}{ An. claviger } & - WORLDCLIM precipitation P2 & - NLST MX & - EVI P2 \\
\hline & - WORLDCLIM precipitation A0 & $\cdot$ MIR MN & $\cdot$ DEM \\
\hline & - Population density & $\cdot$ NLST AO & - NLST MN \\
\hline & - MIR A3 & - WORLDCLIM precipitation P3 & - NLST A2 \\
\hline & - WORLDCLIM precipitation DA & - NLST MN & - CMORPH precipitation A 1 \\
\hline & • EVI D2 & - DLST AO & • MIR D3 \\
\hline & $\cdot$ NLST P3 & $\cdot$ DLST A1 & - WORLDCLIM precipitation D3 \\
\hline & • EVI P2 & - DLST MX & - CMORPH precipitation A2 \\
\hline & $\cdot$ NLST A3 & - NDVI A2 & - NLST AO \\
\hline & - DLST AO & • NDVI VR & - Population density \\
\hline \multirow[t]{10}{*}{ Oc. punctor } & - Population density & - Population density & - NDVI D1 \\
\hline & - MIR P1 & - MIR P1 & • MIR P1 \\
\hline & • EVI P3 & - EVI P3 & • DLST P2 \\
\hline & - NDVI P3 & - NDVI P3 & • EVI P2 \\
\hline & $\cdot$ NDVI P2 & $\cdot$ NDVI P2 & - MIR A3 \\
\hline & - DLST MN & - DLST MN & - WORLDCLIM precipitation A3 \\
\hline & - DEM & - DEM & - NDVI A3 \\
\hline & - CMORPH precipitation A2 & - CMORPH precipitation A2 & - WORLDCLIM P3 \\
\hline & - CMORPH precipitation A1 & - CMORPH precipitation A1 & - EVI MN \\
\hline & - WORLDCLIM precipitation P3 & - WORLDCLIM precipitation P3 & - CMORPH precipitation A2 \\
\hline
\end{tabular}


Table 2 Accuracy measures for the environmental suitability per species and per model

\begin{tabular}{|c|c|c|c|c|}
\hline SPECIES & & NLDA & $\mathrm{RF}$ & GLM \\
\hline \multirow[t]{2}{*}{ Cs. annulata } & Specificity (Cl) & $0.805(0.596-0.884)$ & $\mathbf{0 . 8 9 2}(0.808-0.936)$ & $0.576(0.442-0.811)$ \\
\hline & Sensitivity $(\mathrm{Cl})$ & $0.639(0.541-0.829)$ & $0.637(0.696-0.779)$ & $\mathbf{0 . 7 5 3}(0.498-0.868)$ \\
\hline \multirow[t]{2}{*}{ An. claviger } & Specificity (Cl) & $0.670(0.559-0.850)$ & $0.908(0.875-0.944)$ & $0.652(0.452-0.820)$ \\
\hline & Sensitivity $(\mathrm{Cl})$ & $0.772(0.567-0.866)$ & $\mathbf{0 . 8 9 0}(0.827-0.945)$ & $0.709(0.512-0.890)$ \\
\hline \multirow[t]{2}{*}{ Oc. punctor } & Specificity (Cl) & $0.828(0.735-0.954)$ & $\mathbf{0 . 9 1 0}(0.825-0.944)$ & $0.765(0.574-0.828)$ \\
\hline & Sensitivity (Cl) & $0.932(0.795-1.00)$ & $0.945(0.890-1.00)$ & $0.808(0.685-0.945)$ \\
\hline
\end{tabular}

The confidence intervals $(\mathrm{Cl})$ are based on 2000 stratified bootstrap replicates.

The best values for sensitivity and specificity for each species are printed in bold.

environmental data and applying three different modelling approaches, i.e., NLDA, RF and GLM. When comparing the maps for each species produced with the three different modelling techniques, we see consistent spatial patterns, but different levels of predicted environmental suitability. The average predicted environmental suitability was lower for NLDA than for the other methods. This is visible in the predominance of blue colours in the NLDA maps.

Most of the variables highlighted by the models as important are in agreement with field experience, existing biological knowledge, and known habitat preference of these species in Belgium (MODIRISK) [1]. Precipitation and temperature for Cs. annulata and An. claviger are important in both our study and MODIRISK. For $A n$. claviger the population density is also reported as important, both in MODIRISK and in our study (for NLDA and GLM). Both studies show a preference of the latter species for the coastal area. The GLM map for An. claviger shows wetland and the floodplains of the big rivers as suitable environments, in accordance with field knowledge. Oc. punctor occurrence is related to land surface temperature and particularly with vegetation indices, in the Netherlands as well as in Belgium. This species is generally found in forests and natural areas. Population density is recorded in our study as one of the most influential variables in NLDA and RF models for Oc. punctor and Cs. annulata. Cs. annulata is known to breed in a wide variety of habitats and to be associated with areas with human activity [5], whereas Oc. punctor prefers swampy forest with boggy waters and seldom flies out of the forest [5], characteristics that suggest a negative relationship with human presence.

In terms of model performance, RF shows the best discrimination skills. Also in other studies, this technique was consistently reported to outperform other traditional

Table 3 Number of presence and absence points per species

\begin{tabular}{lll}
\hline SPECIES & Presence & Absence \\
\hline C. annulata & 438 & 344 \\
An. claviger & 127 & 655 \\
Oc. punctor & 73 & 709 \\
\hline
\end{tabular}

modelling techniques [6,7]. Only the GLM for Cs. annulata has a higher sensitivity than RF. Random forest sensitivity and specificity are excellent, often equal to or larger than 0.9. However, if we want to interpret these values, we have to consider that the training data are also used to evaluate the model, meaning that the accuracy measures will be overestimated $[8,9]$. Although most modellers consider that external validation is preferable to internal, there are cases where internal validation (i.e., the model ability to fit the training data) is sufficient. If the goal is to describe a pattern, overestimating the accuracy is not a problem. This is the case for models seeking to convert the observed records of a species into a suitability score [10], as in our study. Overall, when making predictions based on occurrence data, presence data are more reliable than absence data [11]. Absence points may represent areas where the trap failed to catch a mosquito despite these mosquitoes being present in the area, or areas that are in principle suitable, but which have not yet been invaded. Therefore, in these cases, it is recommended to prioritize the sensitivity over the specificity [12].

Sensitivity and specificity measures were used to compare techniques for the same species and in the same geographical area. It was not possible to compare model performance between species because the traditional methods are highly influenced by the relative areas of occurrence of different species and by the geographical extent: increasing the geographical extent outside the presence environmental domain leads to a larger score for the area under the curve [13]. In fact, it has been shown that the relative occurrence area of the species influences the results of the evaluation scores, implying that models of rare species with high environmental specificity will yield to higher discrimination values $[14,15]$ and that species with restricted environmental tolerance and/or distributions are usually reported to be well predicted [16]. This is indeed what we would observe if we compared sensitivity and specificity between species: Oc. punctor is mainly observed in the east of the country and the random forest has the highest discrimination skills, compared to other species.

To create a reliable model, it is generally considered necessary to have the same number of presence and 
absence points as input. This is because having a different number will create a bias in the model prediction towards the more prevalent category (presence or absence) [17]. For NLDA and GLM, this balancing, i.e., considering the same number of presences and absences, is accomplished at the bootstrapping stage. However, for techniques such as random forest it is necessary to select a 'balanced' subset of the data.

Some areas were excluded from the sampling scheme, because they were deemed unsuitable for mosquitoes. At the modelling stage, this had to be corrected by adding absence points in these unsampled regions (for details, see methods section). In our study, this adjustment was possible because it was known that the unsampled regions would be negative for presence of mosquitoes. Generally speaking, avoiding bias in sampling strategies is more advisable than correcting for lack of data at the modelling stage.

The aim of the study was to investigate the spatial distribution of mosquito species and to compare the performance of three statistical models. In recent years, predictive modelling of species distribution has become an increasingly important tool to address various issues in ecology, biogeography, evolution, conservation biology and climate change research [18]. Beyond describing species distributions, these models have become an important and widely used decision making tool for a variety of biogeographical applications, such as mapping risk of vector-borne disease spread, and determining locations that are potentially susceptible to invasion [19]. Species distribution modelling using flexible machine learning approaches has been successfully applied to quantify and to map the global distribution of hosts [20], disease vectors [21], pathogens [22], and infection and outbreak risk [23].

\section{Conclusions}

In this study we mapped the environmental suitability for three mosquito species with three different modelling techniques. For each species, the models produced consistent spatial patterns, but different levels of prediction ranges. The average predicted environmental suitability was lower for NLDA than for the other methods. The variables selected as important in the models were consistent with field experience and the existing knowledge about these species. All the modelling techniques showed a satisfactory to excellent accuracy; the best accuracy was obtained with the random forest model.

The insights obtained in this study can be used to improve future predictions for vector and non-vector species. The output of this type of distribution modelling methods can be used as input for epidemiological models and can be helpful to identify suitable areas for a given species, at risk of successful invasion if the species is still absent. Such areas may therefore need particular attention in terms of measures of prevention.

\section{Methods}

The input for spatial distribution modelling consists of mosquito field data and environmental, often satellite, data. Here we describe the mosquito data collection, the environmental data used and the statistical methods applied.

\section{Mosquito data}

Data were collected by the Dutch Centre for Monitoring of Vectors during the National Mosquito Survey program, from April to October 2010-2013 [24]. These consisted of mosquito abundance data, sampled at 778 locations. Each of the locations was sampled only once and each trap was active for one week. At the sampled locations, mosquitoes were captured by means of $\mathrm{CO}_{2-}$ baited Mosquito Magnet Liberty Plus MM3100 traps (Woodstream ${ }^{\circ}$ Co., Lititz, USA). These traps have been evaluated successfully for trapping and surveillance against a variety of mosquito genus and species [25,26], and have been used successfully in the national inventory of mosquitoes in Belgium, MODIRISK [2], also to capture Culiseta annulata, Anopheles claviger and Ochlerotatus punctor. For our survey, the traps were randomly located in the Netherlands, following the study design described in Ibañez-Justicia et al. [24]. Of the traps, $40 \%$ were placed in urban areas, $40 \%$ in agricultural areas and $20 \%$ in natural areas. Natural areas were sampled to a lesser extent because of their presumed lower involvement in human and veterinary health risks.

As described in Ibáñez-Justicia et al. [24], high productivity agricultural areas, such as arable land or permanent crops, were not sampled as they are considered to be unsuitable due to a lack of mosquito breeding sites. Therefore, areas with beet, grain, maize, potatoes and other agricultural crops, bulb flowers, productivity orchards and greenhouses were excluded from the sampling. When the goal is to estimate the potential distribution of a species, it is important that absence data come from environmental conditions that are known to be unsuitable for the species [27]. If information on absence is not available, absences can be generated outside the environmental domain where the species is present [14]. This has been done, for example, in Jiménez-Valverde and Lobo [28], where probable absences were randomly selected in the areas having environmental values outside the range of observed presences. In a similar way, forty-three absence points in our study were generated in the land cover types that are deemed unsuitable for mosquitoes. Omitting this information, would have led to unrealistic predictions, as is for example shown in Figure 2. In Figure 2A, the presence and absence points for An. claviger are shown as 


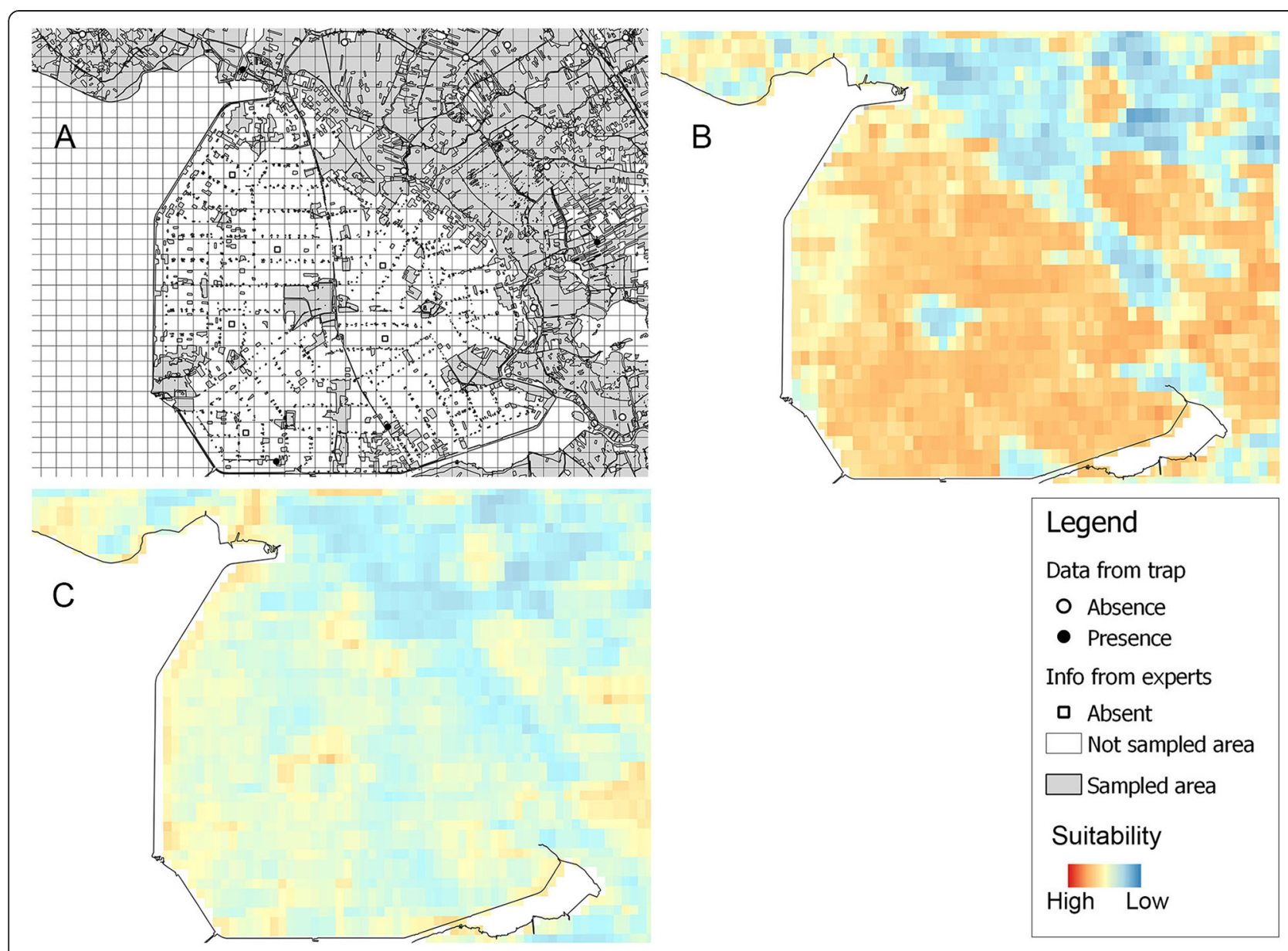

Figure 2 Probable absences added in unsuitable unsampled areas. A - Absence points added to the An. claviger data in part of Flevoland province. The grey area was sampled and the white area was excluded from the sampling because it was considered unsuitable for mosquitoes. White and black circles indicate negative and positive traps, respectively. White squares indicate the probable absences added in unsuitable unsampled areas. B - Random forest predictions for An. claviger without pseudo-absences. Environmental suitability is depicted using a gradient fill: blue indicates low environmental suitability, red indicates high suitability. C - Random forest predictions for An. claviger with pseudo-absences.

black and white circles in an area of Flevoland province (north-west). The grey area was sampled and the white area was excluded from the sampling because it was considered unsuitable for mosquitoes. Only two traps were located in this part of the region, in two fragments that were part of the sampled area, and they were both positive for the presence of mosquitoes. Both positive traps were located in small areas enclaved in pixels that were mainly considered to be unsuitable (intensively used agricultural fields). However, these possibly unsuitable areas were not sampled and therefore there are no data informing the model of their unsuitability. In a model without probable absence added, the two pixels are identified as suitable and consequently the whole area will be predicted as being suitable (Figure 2B), potentially incorrectly. If we introduce also absence points, the model is provided with more complete information and gives more realistic predictions (Figure 2C).
The abundance data were reclassified into data of presence (when at least one mosquito was found in the trap) and absence (when no mosquitoes were found in the trap), because the number of mosquitoes in each location was measured in different weeks and the mosquito abundance is expected to vary seasonally. The resolution used for the maps is $1 \mathrm{~km}^{2}$. When a presence and an absence point were in the same square kilometre only the presence point was selected because presences inform about the places that are environmentally suitable for a species, but absences do not necessarily indicate the opposite [29]. This reduced the number of locations used in the analysis from 778 to 766 .

\section{Environmental variables}

The environmental data included in the analysis as predictor variables are $1 \mathrm{~km}^{2}$ resolution satellite images and meteorological data in raster file format, commonly used 
for mosquito distribution modelling [30]. The images were obtained from the MODIS sensor on NASA's Terra and Aqua satellites [31,32] for 2000-2012 and subjected to temporal Fourier transformation [33,34] to summarise the images and to produce sets of data that capture characteristics of the annual seasonality: the mean, the annual bi-annual and tri-annual amplitudes and phases, the maxima, minima and variances of variances of the middle infra-red (MIR), the daytime Land Surface Temperature (dLST), the night-time Land Surface Temperature (nLST), the Enhanced Vegetation Index (EVI) and the Normalized Difference Vegetation Index (NDVI) signals [35]. Other environmental data used in this study are precipitation (WorldClim [36] and CMORPH [37] 1950-2000), population density (compiled from the Gridded Population of the World Dataset 2000 [38]), the digital elevation model (MODIS [32] 2012) and land cover (Corine land cover map of 2006) [39]. A list of the Fourier components is provided in Table 4 and the environmental data are listed in Table 5. Predictor variables were organized as raster type files and for each trap location the pixel values of the environmental variables were extracted.

\section{Statistical analysis}

Species distribution models quantitatively describe areas that support the presence of a given species, based on known occurrence data and the associated environmental conditions [40]. Here, three methods suitable for occurrence data have been applied, i.e., non-linear discriminant analysis, random forest analysis and a generalised linear model, aimed at describing the relationship between response and predictor variables. For all three modelling techniques, the output was an environmental suitability

Table 4 Fourier components from temporal Fourier analysis of an imagery time series

\begin{tabular}{ll}
\hline Component & Description \\
\hline A0 & Fourier mean for entire time series \\
MX & Minimum value \\
A1 & Maximum value \\
A2 & Amplitude of annual cycle \\
A3 & Amplitude of bi-annual cycle \\
VR & Amplitude of tri-annual cycle \\
P1 & Total variance \\
P2 & Phase of annual cycle \\
P3 & Phase of bi-annual cycle \\
D1 & Phase of tri-annual cycle \\
D2 & Proportion of total variance due to annual cycle \\
D3 & Proportion of total variance due to bi-annual cycle \\
DA & Proportion of total variance due to tri-annual and cycle \\
\hline
\end{tabular}

Component is the name used in the software Vecmap.
Table 5 Environmental predictor variables

\begin{tabular}{ll}
\hline Source & Variable \\
\hline MODIS & Middle infra-red (MIR) \\
MODIS & Day-time land surface temperature (DLST) \\
MODIS & Night-time land surface temperature (NLST) \\
MODIS & Enhanced vegetation index (EVI) \\
MODIS & Normalised difference vegetation index (NDVI) \\
CMORPH & Precipitation \\
WorldClim & Precipitation \\
MODIS & Digital elevation model (DEM) \\
Gridded population & Human population density \\
of the world & \\
European Environment & Corine land cover \\
Agency & \\
\hline
\end{tabular}

indicator for each species, expressed as a value between 0 (low suitability) and 1 (high suitability). The predicted environmental suitability is visualised in maps.

\section{Non-linear discriminant analysis}

Models created using NLDA [34] require presence and absence data to be grouped into clusters based on attribute data. In this way, a discriminant function can be created and predictive maps based on these clusters can be made. The main advantage of the clustering is that it handles spatial heterogeneity of habitat niches and zones. The data were clustered using the k-means clustering algorithm. Since the important variables for the species were unknown, generic variables were used for clustering. These variables were DEM and the means, amplitudes, maxima, minima, variances (of the entire signal) and phases of MIR, LST and NDVI. NLDA models were bootstrapped [41], meaning that 100 models were run and that for each model a sample of an equal number of presence and absence points was taken from the training set with replacement. The final predictions are based on the average of the 100 models.

\section{Random forest}

A random forest [42] method consists of an ensemble of classification and regression trees (CART; [43]) constructed using a random subset of both the available samples and the attributes recorded for each data point. A CART tree is a hierarchical structure that allows a data point to be assigned to a particular class based on its attribute values. For the random forest method it is necessary to have the same number of presence and absence points as input, in order to obtain unbiased model predictions [44]. For techniques such as NLDA and GLM, this balancing is guaranteed at the bootstrapping stage, but this is not the case for RF. Therefore, before the model was run, five 'balanced' subsets of the complete 
dataset were randomly created. For each species, if there were more absence than presence points, all the presence points were used and a random subset of the absences was selected. If the presence points outnumbered the absence points, the procedure was inversed. The RF models were not bootstrapped because inherently RF uses a rationale similar to the bootstrapping approach, being based on several CART trees. The final predictions are the average of the five submodels.

\section{Logistic regression}

For the GLM analysis, a logistic regression model was used because the response is a binary variable (presence/ absence). GLM models can account for spatial autocorrelation by using an autoregressive term or mixture model. The effect of spatial autocorrelation on the training set was checked in the correlograms, i.e., plots of distance between points and the Moran's I index of their correlation. Since the correlation effect was not strong (with the exception at extreme distances where it is known that values for Moran's I may be erratic due to fewer points that can be compared [45]) there was no need to account for spatial autocorrelation. As for NLDA, the GLM models were bootstrapped 100 times with a sample of an equal number of presence and absence points after which the 100 models were averaged to produce the final predictions.

\section{Model evaluation}

The choice of evaluation strategy needs to be explicitly related to the subject and goals of modelling. Here, the aim is to describe a given pattern and get a suitability score. In this context, simple forms of verifications, e.g. the number of false negatives, is appropriate to check whether models are performing as intended [10]. For each model, sensitivity and specificity were calculated, where sensitivity is the ability of a model to correctly identify known positive sites and specificity is the ability of a model to correctly identify known negative sites. Sensitivity and specificity were reported together with the values of their confidence intervals. The confidence intervals are calculated based on 2000 stratified bootstrap replicates at $95 \%$ level. Sensitivity and specificity were used to compare the results produced with NLDA, RF and GLM for the same species. A list of the most important variables used in the models is provided. For NLDA and GLM the top 10 ranked variables are listed and for RF variable importance is given as mean decrease in Gini index [42,43].

The analysis has been performed with the software Vecmap demo version [46]. The accuracy measures have been calculated with R 3.0.2 statistical language environment [47] (R Development Core Team 2013), using of the R-packages pROC, ROCR, OptimalCutpoints.

\section{Abbreviation}

NLDA: Non-linear discriminant analysis; RF: Random forest; GLM: Generalised linear model; Cs.: Culiseta; An.: Anopheles; Oc.: Ochlerotatus; MIR: Middle infra-red; dLST: Daytime Land Surface Temperature; nLST: Night-time Land Surface Temperature; EVI: Enhanced Vegetation Index; NDVI: Normalized Difference Vegetation Index; DEM: Digital Elevation Model.

\section{Competing interests}

The authors declare that they have no competing interests.

\section{Authors' contributions}

DC, NH and AIJ designed the study. AIJ collected the data. DC did the data preparation and performed the statistical analysis. DC, NH and AIJ wrote the paper. All authors read and approved the final version of the manuscript.

\section{Acknowledgements}

The authors would like to thank David Morley, Els Ducheyne and Veerle Versteirt for their help with Vecmap ${ }^{\text {TM }}$ and Hans Heesterbeek and the FAH writing club for their helpful comments on the manuscript. The authors would also like to thank their recently deceased beloved colleague Dr. Ernst-Jan Scholte for promoting the idea of modelling the CMV mosquito surveillance data in the Netherlands. This study was funded by EU grant FP7-261504 EDENext and is catalogued by the EDENext Steering Committee as EDENext243 (http://www. edenext.eu). The contents of this publication are the sole responsibility of the authors and do not necessarily reflect the views of the European Commission.

\section{Author details}

${ }^{1}$ Faculty of Veterinary Medicine, Utrecht University, Utrecht, The Netherlands. ${ }^{2}$ Centre for Monitoring of Vectors, Food and Consumer Product Safety Authority, Wageningen, The Netherlands.

Received: 16 December 2014 Accepted: 31 January 2015

Published online: 27 February 2015

\section{References}

1. Versteirt V, De Clercq E, Dekoninck W, Damiens D, Ayrinhac A, Jacobs F, et al. Mosquito Vectors of Disease: Spatial Biodiversity, Drivers of Change, and Risk. Final Report. Brussels: Belgian Science Policy; 2009.

2. Versteirt V, Boyer S, Damiens D, De Clercq EM, Dekoninck W, Ducheyne E, et al. Nationwide inventory of mosquito biodiversity (Diptera: Culicidae) in Belgium, Europe. Bull Entomol Res. 2013;103:193-203.

3. Rogers DJ. Models for vectors and vector-borne diseases. Adv Parasitol. 2006;62:1-35.

4. Elith J, Graham CH, Anderson RP, Dudík M, Ferrier S, Guisan A, et al. Novel methods improve prediction of species' distributions from occurrence data. Ecography (Cop). 2006;29:129-51.

5. Becker N, Petric D, Zgomba M, Boase C, Madon M, Dahl C, et al. Mosquitoes and Their Control. Heidelberg, Dordrecht, New York: Springer; 2010. p. 577.

6. Cutler DR, Edwards Jr TC, Beard KH, Cutler A, Hess KT, Gibson J, et al. Random forests for classification in ecology. Ecology. 2007;88:2783-92.

7. Peters J, De Baets B, Verhoest NEC, Samson R, Degroeve S, De BP, et al. Random forests as a tool for ecohydrological distribution modelling. Ecol Modell. 2007;207:304-18.

8. Harrell FE, Lee KL, Mark DB. Tutorial in biostatistics multivariable prognostic models: issues in developing models, evaluating assumptions and adequacy, and measuring and reducing errors. Stat Med. 1996;15:361-87.

9. Hastie T, Tibshirani R, Friedman J, Franklin J. The elements of statistical learning: data mining, inference and prediction. Math Intell. 2005;27:83-5.

10. Araujo MB, Guisan A. Five (or so) challenges for species distribution modelling. J Biogeogr. 2006;33:1677-88.

11. Dicko AH, Lancelot R, Seck MT, Guerrini L, Sall B, Lo M, et al. Using species distribution models to optimize vector control in the framework of the tsetse eradication campaign in Senegal. Proc Natl Acad Sci. 2014;111:10149-54.

12. De Clercq EM, Leta S, Estrada-Peña A, Madder M, Adehan S, Vanwambeke SO. Species distribution modelling for Rhipicephalus microplus (Acari: Ixodidae) in Benin, West Africa: Comparing datasets and modelling algorithms. Prev Vet Med. 2015;118:8-21.

13. Lobo JM, Jiménez-Valverde A, Real R. AUC: a misleading measure of the performance of predictive distribution models. Glob Ecol Biogeogr. 2008;17:145-51. 
14. Jiménez-Valverde A, Lobo JM, Hortal J. Not as good as they seem: the importance of concepts in species distribution modelling. Divers Distrib. 2008;14:885-90.

15. McPherson J, Jetz W. Effects of species' ecology on the accuracy of distribution models. Ecography (Cop). 2007;30:135-51.

16. Tsoar A, Allouche O, Steinitz O, Rotem D, Kadmon R. A comparative evaluation of presence-only methods for modelling species distribution. Divers Distrib. 2007;13:397-405.

17. Fielding $\mathrm{AH}$, Bell JF. A review of methods for the assessment of prediction errors in conservation presence/absence models. Environ Conserv. 1997;24:38-49.

18. Guisan A, Thuiller W. Predicting species distribution: offering more than simple habitat models. Ecol Lett. 2005;8:993-1009.

19. Miller J. Species distribution modeling. Geogr Compass. 2010;4:490-509.

20. Alexander NS, Morley D, Medlock J, Searle K, Wint W. A first attempt at modelling roe deer (Capreolus capreolus) distributions over Europe. Open Heal Data. 2014;2:e2

21. Sinka ME, Bangs MJ, Manguin S, Rubio-Palis Y, Chareonviriyaphap T, Coetzee $M$, et al. A global map of dominant malaria vectors. Parasit Vectors. 2012;5:69.

22. Bhatt $\mathrm{S}$, Gething PW, Brady OJ, Messina JP, Farlow AW, Moyes $\mathrm{CL}$, et al. The global distribution and burden of dengue. Nature. 2013;496:504-7.

23. Fischer EAJ, Boender G-J, Nodelijk G, de Koeijer AA, Van Roermund HJ. The transmission potential of Rift Valley fever virus among livestock in the Netherlands: a modelling study. Vet Res. 2013:44:58.

24. Ibañez-Justicia A, Stroo A, Dik M, Beeuwkes J, Scholte EJ. National Mosquito (Diptera, Culicidae) Survey in the Netherlands 2010-2013. J Med Entomol 2015. doi: 10.1093/jme/tju058

25. Dennett JA, Vessey NY, Parsons RE. A comparison of seven traps used for collection of Aedes albopictus and Aedes aegypti originating from a large tire repository in Harris County (Houston), Texas. J Am Mosq Control Assoc. 2004;20:342-9.

26. McKenzie KE, Bedard SD. A Comparison of the Efficacy of Several Models of Mosquito Magnet Traps Baited with Lurex 3 versus Commercially Available Traps Supplied with Octenol on the Collection of Mosquitoes in Oahu. Hawaii: Primarily Aedes albopictus; 2004.

27. Chefaoui RM, Lobo JM. Assessing the effects of pseudo-absences on predictive distribution model performance. Ecol Modell. 2008;210:478-86.

28. Jiménez-Valverde A, Lobo JM. Potential distribution of the endangered spider Macrothele calpeiana (Walckenaer, 1805)(Araneae, Hexathelidae) and the impact of climate warming. Acta Zool Sin. 2007;53:865-76.

29. Lobo JM, Jiménez-Valverde A, Hortal J. The uncertain nature of absences and their importance in species distribution modelling. Ecography (Cop) 2010;33:103-14.

30. European Centre for Disease Prevention and Control (ECDC). Environmental risk mapping: Aedes albopictus in Europe. Stockholm: ECDC; 2013. Available from: http://www.ecdc.europa.eu/en/publications/Publications/climatechange-environmental-risk-mapping-aedes.pdf.

31. Justice C, Townshend J, Vermote E, Sohlberg R, Descloitres J, Roy D, et al. Preliminary land surface products from the NASA Moderate Resolution Imaging Spectroradiometer (MODIS). In: Geosci Remote Sens Symp 2000 Proceedings IGARSS 2000 IEEE 2000 Int. Volume 3. 2000. p. 1157-62.

32. MODIS [modis.gsfc.nasa.gov]

33. Rogers DJ, Williams BG. Tsetse distribution in Africa: seeing the wood and the trees. 1994.

34. Rogers DJ. Satellites, space, time and the African trypanosomiases. Adv Parasitol. 2000:47:129-71.

35. Scharlemann JPW, Benz D, Hay SI, Purse BV, Tatem AJ, Wint GRW, et al. Global data for ecology and epidemiology: a novel algorithm for temporal Fourier processing MODIS data. PLoS One. 2008;3:e1408.

36. WorldClim - Global Climate Data [www.worldclim.org]

37. National Weather Service Climate Prediction Center [www.cpc.ncep.noaa.gov]

38. Socioeconomic Data and Applications Center [sedac.ciesin.columbia.edu]

39. European Environment Agency [www.eea.europa.eu]

40. Elith J, Leathwick JR. Species distribution models: ecological explanation and prediction across space and time. Annu Rev Ecol Evol Syst. 2009;40:677-97.

41. Efron B, Tibshirani RJ. An Introduction to the Bootstrap. London, UK: Chapman and Hall; 1993

42. Breiman L. Random forests. Mach Learn. 2001;45:5-32.
43. Breiman L, Friedman JH, Olshen RA, Stone CJ. Classification and Regression Trees. Belmont, California, USA: Wadsworth international group; 1984.

44. McPherson J, Jetz W, Rogers DJ. The effects of species' range sizes on the accuracy of distribution models: ecological phenomenon or statistical artefact? J Appl Ecol. 2004;41:811-23.

45. de Jong P, Sprenger C, Veen F. On extreme values of Moran's I and Geary's C. Geogr Anal. 1984;16:17-24.

46. Avia-GIS: Vecmap ${ }^{\mathrm{TM}}$ : A one-stop-shop for vector mapping, Zoersel, Belgium. http://www.avia-gis.com/vecmap. 2014.

47. R Development Core Team. R: A Language and Environment for Statistical Computing. 2013.

\section{Submit your next manuscript to BioMed Central and take full advantage of:}

- Convenient online submission

- Thorough peer review

- No space constraints or color figure charges

- Immediate publication on acceptance

- Inclusion in PubMed, CAS, Scopus and Google Scholar

- Research which is freely available for redistribution 